\title{
Weighted Hölder Continuity of Hyperbolic Harmonic Bloch functions
}

\author{
Guangbin Ren and U. Kähler
}

\begin{abstract}
Characterizations of weighted Hölder continuity and weighted Lipschitz continuity are obtained for the hyperbolic Bloch functions on the unit ball of $\mathbb{R}^{n}$. Similar results are extended to hyperbolic little Bloch and Besov spaces.
\end{abstract}

Keywords: Hyperbolic Bloch space, hyperbolic Besov spaces, invariant Laplacian

AMS subject classification: Primary 42A35, secondary 32A50

\section{Introduction}

Let $\mathbb{B}$ be the unit ball in $\mathbb{R}^{n}$ with $n \geq 2, d \nu$ the normalized measure on $\mathbb{B}$ and $d \sigma$ the normalized surface measure on the unit sphere $S=\partial \mathbb{B}$. We shall consider the Poincaré metric in $\mathbb{B}$

$$
d s^{2}=\frac{|d x|^{2}}{\left(1-|x|^{2}\right)^{2}} .
$$

The corresponding Laplace-Beltrami operator and gradient are given by

$$
\begin{aligned}
& \widetilde{\triangle} f(x)=\left(1-|x|^{2}\right)^{2}\left(\triangle f(x)+\frac{2(n-2)}{1-|x|^{2}} \sum_{i=1}^{n} x_{i} \frac{\partial f}{\partial x_{i}}(x)\right), \\
& \widetilde{\nabla} f(x)=\left(1-|x|^{2}\right) \nabla f(x),
\end{aligned}
$$

where $\triangle$ and $\nabla$ denote the usual Laplacian and gradient, respectively. They are invariant in the sense

$$
\begin{aligned}
& \widetilde{\triangle} f(x)=\triangle\left(f \circ \varphi_{x}\right)(0), \\
& \widetilde{\nabla} f(x)=\nabla\left(f \circ \varphi_{x}\right)(0),
\end{aligned}
$$

where the Möbius transformation $\varphi_{x} \in \operatorname{Aut}(B), x \in \mathbb{B}$, is an involutionary automorphism of $\mathbb{B}$ with $\varphi_{x}(0)=x$. Notice that for any $f \in C^{2}(\mathbb{B})$

$$
|\widetilde{\nabla} f(x)|=\left(1-|x|^{2}\right)|\nabla f(x)| \text {. }
$$

Guangbin Ren: Univ. of Sci. \& Techn. of China, Dept. Math., Hefei 230026, P.R. China and Univ. of Aveiro, Dept. Math., 3810-193 Aveiro, Portugal

U. Kähler: Univ. of Aveiro, Dept. Math., 3810-193 Aveiro, Portugal; uwek@mat.ua.pt Project supported by the NNSF of China (No. 10001030, 19871081) and the Post-doctoral Fellowship of University of Aveiro, UID "Mathematics and Applications". 
A function $f \in C^{2}(\mathbb{B})$ is called hyperbolic harmonic or simply $\mathcal{H}$-harmonic if it is annihilated by the invariant Laplacian on $\mathbb{B}$. The

- $\mathcal{H}$-harmonic Bloch space $\mathcal{B}$ is the space of all $\mathcal{H}$-harmonic functions on $\mathbb{B}$ for which $\sup _{x \in \mathbb{B}}|\widetilde{\nabla} f(x)|<\infty$

- $\mathcal{H}$-harmonic little Bloch space $\mathcal{B}_{0}$ consists of all functions $f \in \mathcal{B}$ such that $\lim _{|x| \rightarrow 1}|\widetilde{\nabla} f(x)|=0$

- $\mathcal{H}$-harmonic Besov space $\mathcal{B}_{p}$ is the space of all -harmonic functions on $B$ for which $\int_{\mathbb{B}}|\widetilde{\nabla} f(x)|^{p} d \tau(x)<\infty$ where $d \tau(x)=\left(1-|x|^{2}\right)^{-n} d \nu(x)$ is the invariant measure on $\mathbb{B}$.

Let $\alpha, \beta \geq 0$ and $0<\lambda<1$, and let $f$ be a continuous function in $\mathbb{B}$. If there exist a constant $C$ such that

$$
\left(1-|x|^{2}\right)^{\alpha}\left(1-|y|^{2}\right)^{\beta}|f(x)-f(y)| \leq C|x-y|
$$

for any $x, y \in \mathbb{B}$, then we say that $f$ satisfies a weighted Lipschitz condition of indices $(\alpha, \beta)$. If there exist a constant $C$ such that

$$
\left(1-|x|^{2}\right)^{\alpha}\left(1-|y|^{2}\right)^{\beta}|f(x)-f(y)| \leq C|x-y|^{\lambda}
$$

for any $x, y \in \mathbb{B}$, then we say that $f$ satisfies a weighted Hölder condition of indices $(\alpha, \beta, \lambda)$.

The main purpose of this paper is to give some characterizations of $\mathcal{B}, \mathcal{B}_{0}$ and $\mathcal{B}_{p}$ in terms of weighted Hölder or Lipschitz conditions. We refer to $[3,4,7,8]$ for corresponding results in the complex unit ball for holomorphic or $\mathcal{M}$-harmonic functions. See $[6,9,12,13,15,16]$ for various characterization of the Bloch, little Bloch, and Besov spaces in the unit ball of $\mathbb{C}^{n}$.

Our main results are the following three theorems.

Theorem 1.1. Let $f$ be a hyperbolic harmonic function on $\mathbb{B}$. Then the following statements are equivalent:

(i) $f \in \mathcal{B}$.

(ii) $f$ satisfies a weighted Lipschitz condition of indices $(\alpha, \beta)$ with $\alpha+\beta=1$, $\alpha, \beta>0$.

(iii) $f$ satisfies a weighted Hölder condition of indices $(\alpha, \beta, \lambda)$ with $\alpha+\beta=\lambda$, $\alpha, \beta>0$ and $0<\lambda<1$.

Theorem 1.2. Let $0<\lambda<1$ and $\alpha, \beta>0$ with $\alpha+\beta=\lambda$. For any hyperbolic harmonic function $f$ on $\mathbb{B}, f \in \mathcal{B}_{0}$ if and only if

$$
\lim _{|x| \rightarrow 1^{-}} \sup \left\{\left(1-|x|^{2}\right)^{\alpha}\left(1-|y|^{2}\right)^{\beta} \frac{|f(x)-f(y)|}{|x-y|^{\lambda}}: y \in \mathbb{B}, y \neq x\right\}=0 .
$$

Theorem 1.3. Let $p \in(2(n-1), \infty)$. For any hyperbolic harmonic function $f$ on $\mathbb{B}, f \in \mathcal{B}_{p}$ if and only if

$$
\int_{\mathbb{B}} \int_{\mathbb{B}}\left(1-|x|^{2}\right)^{\frac{p}{2}}\left(1-|y|^{2}\right)^{\frac{p}{2}}\left(\frac{|f(x)-f(y)|}{|x-y|}\right)^{p} d \tau(x) d \tau(y)<\infty .
$$




\section{Preliminaries}

We shall be using the following notation: for $x, y \in \mathbb{R}^{n}$ we write in polar coordinates $x=|x| x^{\prime}$ and $y=|y| y^{\prime}$. For any $y, w \in \mathbb{R}^{n}$ the symmetric lemma (see [2: p. 10]) shows

$$
|| y\left|w-y^{\prime}\right|=|| w\left|y-w^{\prime}\right| \text {. }
$$

The same deduction yields

$$
|| y\left|w-\left(1-|w|^{2}\right) y^{\prime}\right|=|| w\left|y-\left(1-|w|^{2}\right) w^{\prime}\right|
$$

so that

$$
\left.|| y\right|^{2} w-\left(1-|w|^{2}\right) y|=| y|||w| y-\left(1-|w|^{2}\right) w^{\prime} \mid .
$$

For any $a \in \mathbb{B}$ we denote the Möbius transformation in $\mathbb{B}$ by $\varphi_{a}$. It is an involutionary automorphism of $\mathbb{B}$ such that $\varphi_{a}(0)=a$ and $\varphi_{a}(a)=0$, which is of the form (see [1: p.25])

$$
\varphi_{a}(x)=\frac{|x-a|^{2} a-\left(1-|a|^{2}\right)(x-a)}{|| x\left|a-x^{\prime}\right|^{2}} \quad(a, x \in \mathbb{B}) .
$$

From (2.2) with $w=a$ and $y=x-a$ we have

$$
\left|\varphi_{a}(x)\right|=\frac{|x-a|}{|| a\left|x-a^{\prime}\right|}
$$

such that

$$
1-\left|\varphi_{a}(x)\right|^{2}=\frac{\left(1-|x|^{2}\right)\left(1-|a|^{2}\right)}{|| a\left|x-a^{\prime}\right|^{2}} .
$$

For any $a \in \mathbb{B}$ and $\delta \in(0,1)$ we denote

$$
\begin{aligned}
& E(a, \delta)=\left\{x \in \mathbb{B}:\left|\varphi_{a}(x)\right|<\delta\right\}, \\
& B(a, \delta)=\{x \in \mathbb{B}:|x-a|<\delta\} .
\end{aligned}
$$

Clearly, $E(a, \delta)=\varphi_{a}(B(0, \delta))$.

Lemma 2.1. Let $x, w \in \mathbb{B}$ and $y \in E(w, \delta)$. Then

$$
\frac{1-\delta}{1+\delta}|| x\left|w-x^{\prime}\right| \leq|| x\left|y-x^{\prime}\right| \leq \frac{1+\delta}{1-\delta}|| x\left|w-x^{\prime}\right|
$$

Proof. From (2.4) and (2.1) we have $\left|\varphi_{y}(w)\right|=\left|\varphi_{w}(y)\right|$, so that $y \in E(w, \delta)$ is equivalent to $w \in E(y, \delta)$. By symmetry, we need only to prove the right inequality. Since

$$
|| x\left|y-x^{\prime}\right| \leq|| x|(y-w)|+|| x\left|w-x^{\prime}\right|
$$

it is enough to show

$$
|y-w| \leq \frac{2 \delta}{1-\delta}|| x\left|w-x^{\prime}\right|
$$

for any $y \in E(w, \delta)$. Denoting $\eta=\varphi_{w}(y)$ we have $y=\varphi_{w}(\eta)$ and $|\eta|<\delta$. From (2.3), a direct computation yields

$$
\left|\varphi_{w}(\eta)-w\right|=\frac{|\eta|}{|| w\left|\eta-w^{\prime}\right|}\left(1-|w|^{2}\right) .
$$

Therefore, by the simple inequality $1-|w| \leq|| x\left|w-x^{\prime}\right|$ we get

as desired

$$
|y-w|=\left|\varphi_{w}(\eta)-w\right| \leq \frac{\delta}{1-\delta}\left(1-|w|^{2}\right) \leq \frac{\delta}{1-\delta} 2|| x\left|w-x^{\prime}\right|
$$


As a direct corollary, we have

$$
1-|x|^{2} \simeq 1-|y|^{2} \quad(x \in E(y, \delta)) .
$$

In fact, taking $w=x$ in Lemma 2.1 we get ||$x\left|y-x^{\prime}\right| \simeq 1-|y|^{2}$. The assertion now follows from (2.1).

Let $F$ be the hypergeometric function (see $[5,10]$ )

$$
F(a, b ; c ; s)=\sum_{k=0}^{\infty} \frac{(a)_{k}(b)_{k}}{k !(c)_{k}} s^{k}
$$

for $a, b, c \in \mathbb{R}$ and $c$ neither zero nor a negative integer, where $(a)_{k}$ denotes the Pochhammer symbol with $(a)_{0}=1$ and $(a)_{k}=a(a+1) \cdots(a+k-1), k \in \mathbb{N}$. These functions have some well-known properties:

(i) Bateman's integral formula

$$
F(a, b ; c+\mu ; s)=\frac{\Gamma(c+\mu)}{\Gamma(c) \Gamma(\mu)} \int_{0}^{1} t^{c-1}(1-t)^{\mu-1} F(a, b ; c ; t s) d t
$$

with $c, \mu>0$ and $s \in(-1,1)$.

(ii) For any integer $m$ [12: p. 69]

$$
\begin{aligned}
F(-m, b ; c ; 1) & =\frac{(c-b)_{m}}{(c)_{m}} \\
F(-m, a+m ; c ; 1) & =\frac{(-1)^{m}(1+a-c)_{m}}{(c)_{m}} .
\end{aligned}
$$

The following identity furnishes the hypergeometric function with an integral representation.

Lemma 2.2. Let $t>1, \lambda \in \mathbb{R}$ and $r \in(-1,1)$. Then

$$
\int_{-1}^{1} \frac{\left(1-u^{2}\right)^{(t-3) / 2}}{\left(1-2 r u+r^{2}\right)^{\lambda}} d u=\frac{\Gamma\left(\frac{t-1}{2}\right) \Gamma\left(\frac{1}{2}\right)}{\Gamma\left(\frac{t}{2}\right)} F\left(\lambda, \lambda+1-\frac{t}{2} ; \frac{t}{2} ; r^{2}\right) .
$$

Proof. Let $C_{m}^{\lambda}$ be the Gegenbauer polynomials. These polynomials can be defined by the generating function

$$
\left(1-2 r u+r^{2}\right)^{-\lambda}=\sum_{m=0}^{\infty} C_{m}^{\lambda}(u) r^{m}
$$

where

$$
\begin{aligned}
C_{2 m}^{\lambda}(u) & =(-1)^{m} \frac{(\lambda)_{m}}{m !} F\left(-m, m+\lambda ; \frac{1}{2} ; u^{2}\right) \\
C_{2 m+1}^{\lambda}(u) & =(-1)^{m} \frac{(\lambda)_{m}}{m !} 2 u F\left(-m, m+\lambda+1 ; \frac{3}{2} ; u^{2}\right) .
\end{aligned}
$$

To calculate the integral in (2.9), we apply (2.10) and (2.11) and can deduce that it is only left to evaluate the integral

$$
\int_{-1}^{1}\left(1-u^{2}\right)^{(t-3) / 2} F\left(-m, m+\lambda ; \frac{1}{2} ; u^{2}\right) d u
$$

or rather an integral over the interval $(0,1)$ by the simple change of variables $t=u^{2}$. For this integral, we first use Bateman's integral formula (2.7) with $s=1$, then we apply (2.8) so that it can be represented by Pochhammer symbols. The calculation of integral (2.9) leads to a series which by definition is the desired hypergeometric function 
Lemma 2.3. Let $\alpha>-1$ and $\beta \in \mathbb{R}$. Then for any $x \in \mathbb{B}$

$$
\int_{\mathbb{B}} \frac{\left(1-|y|^{2}\right)^{\alpha}}{|| x\left|y-x^{\prime}\right|^{n+\alpha+\beta}} d \nu(y) \approx \begin{cases}\left(1-|x|^{2}\right)^{-\beta} & \text { if } \beta>0 \\ \log \frac{1}{1-|x|^{2}} & \text { if } \beta=0 \\ 1 & \text { if } \beta<0\end{cases}
$$

where $a(x) \approx b(x)$ means the ratio $\frac{a(x)}{b(x)}$ has a positive finite limit as $|x| \rightarrow 1$.

Proof. Denote the above integral by $J_{\alpha, \beta}(x)$. From Stirling's formula we need only to show

$$
J_{\alpha, \beta}(x)=\frac{\Gamma\left(\frac{n}{2}+1\right) \Gamma(\alpha+1)}{\Gamma\left(\alpha+\frac{n}{2}+1\right)} F\left(\frac{n+\alpha+\beta}{2}, \frac{2+\alpha+\beta}{2} ; \alpha+\frac{n}{2}+1 ;|x|^{2}\right) .
$$

For any continuous function $f$ of one variable and any $\eta \in \partial \mathbb{B}$, we have the formula (see [2: p. 216])

$$
\int_{\partial \mathbb{B}} f(\langle\zeta, \eta\rangle) d \sigma(\zeta)=\frac{\Gamma\left(\frac{n}{2}\right)}{\Gamma\left(\frac{n-1}{2}\right) \Gamma\left(\frac{1}{2}\right)} \int_{-1}^{1}\left(1-u^{2}\right)^{\frac{n-3}{2}} f(u) d u
$$

where $\langle\zeta, \eta\rangle$ stands for the inner product in $\mathbb{R}^{n}$. Taking

$$
f(u)=\left(1-2 r u+r^{2}\right)^{-\frac{n+\alpha+\beta}{2}} \quad(r \in(0,1) \text { fixed })
$$

and combining it with Lemma 2.2 we get

$$
\begin{aligned}
\int_{\partial \mathbb{B}} & \left(1-2 r\langle\zeta, \eta\rangle+r^{2}\right)^{-\frac{n+\alpha+\beta}{2}} d \sigma(\zeta) \\
& =\frac{\Gamma\left(\frac{n}{2}\right)}{\Gamma\left(\frac{n-1}{2}\right) \Gamma\left(\frac{1}{2}\right)} \int_{-1}^{1} \frac{\left(1-u^{2}\right)^{\frac{n-3}{2}}}{\left(1-2 r u+r^{2}\right)^{\frac{n+\alpha+\beta}{2}}} d u \\
& =F\left(\frac{n+\alpha+\beta}{2}, \frac{2+\alpha+\beta}{2} ; \frac{n}{2} ; r^{2}\right) .
\end{aligned}
$$

Consequently, from the polar coordinates formula we get

$$
\begin{aligned}
J_{\alpha, \beta}(x) & =n \int_{0}^{1} r^{n-1}\left(1-r^{2}\right)^{\alpha} d r \int_{S}\left(1-2 r|x|\left\langle x^{\prime}, \zeta\right\rangle+r^{2}|x|^{2}\right)^{-\frac{n+\alpha+\beta}{2}} d \sigma(\zeta) \\
& =C \int_{0}^{1} r^{n-1}\left(1-r^{2}\right)^{\alpha} F\left(\frac{n+\alpha+\beta}{2}, \frac{2+\alpha+\beta}{2} ; \frac{n}{2} ; r^{2}|x|^{2}\right) d r
\end{aligned}
$$

The assertion now follows from Bateman's integral formula (2.7) 


\section{Bloch space}

In this section we give the proof of Theorems 1.1 and 1.2. Theorem 1.1 can be rephrased as the following

Theorem 3.1. Let $0<\alpha<\lambda \leq 1$. For any hyperbolic harmonic function $f$ on $\mathbb{B}$, $f \in \mathcal{B}$ if and only if

$$
\sup \left\{\left(1-|x|^{2}\right)^{\alpha}\left(1-|y|^{2}\right)^{\lambda-\alpha} \frac{|f(x)-f(y)|}{|x-y|^{\lambda}}: x, y \in \mathbb{B}, x \neq y\right\}<\infty .
$$

Proof. We may assume $\alpha \leq \frac{\lambda}{2}$, since one of the indices $\alpha$ and $\lambda-\alpha$ is no greater than $\frac{\lambda}{2}$.

First, let us assume that $f \in \mathcal{B}$. For any $a \in \mathbb{B}$ we have

$$
f(a)-f(0)=\int_{0}^{1} \frac{d f}{d t}(t a) d t=\sum_{k=1}^{n} a_{k} \int_{0}^{1} \frac{\partial f}{\partial x_{k}}(t a) d t
$$

so that

$$
|f(a)-f(0)| \leq n\|f\|_{\mathcal{B}} \int_{0}^{1} \frac{|a|}{1-t^{2}|a|^{2}} d t=\frac{n}{2}\|f\|_{\mathcal{B}} \log \frac{1+|a|}{1-|a|} .
$$

Now, replacing $f$ by $f \circ \varphi_{y}$ and substituting $x=\varphi_{y}(a)$ we get

$$
|f(x)-f(y)| \leq \frac{n}{2}\|f\|_{\mathcal{B}} \log \frac{1+\left|\varphi_{y}(x)\right|}{1-\left|\varphi_{y}(x)\right|}
$$

To estimate the last factor, we can apply the fact that

$$
\log \frac{1+|a|}{1-|a|}=2|a| \sum_{n=0}^{\infty} \frac{|a|^{2 n}}{2 n+1} \leq C|a| \sum_{n=0}^{\infty} \frac{\Gamma(n+\alpha)}{n ! \Gamma(\alpha)}|a|^{2 \alpha}=C \frac{|a|}{\left(1-|a|^{2}\right)^{\alpha}}
$$

for any $0<\alpha<1$ and $a \in \mathbb{B}$. Now, from identities (2.4) - (2.5), we get

$$
\begin{aligned}
\log \frac{1+\left|\varphi_{y}(x)\right|}{1-\mid \varphi_{y}(x)} & \leq C \frac{\left|\varphi_{y}(x)\right|}{\left(1-\left|\varphi_{y}(x)\right|^{2}\right)^{\alpha}} \\
& \leq C \frac{\left|\varphi_{y}(x)\right|^{\lambda}}{\left(1-\left|\varphi_{y}(x)\right|^{2}\right)^{\alpha}} \\
& =C \frac{|x-y|^{\lambda}}{\left(1-|x|^{2}\right)^{\alpha}\left(1-|y|^{2}\right)^{\lambda-\alpha}}\left(\frac{1-|y|^{2}}{\| x\left|y-x^{\prime}\right|}\right)^{\lambda-2 \alpha} \\
& \leq C \frac{2^{\lambda-2 \alpha}|x-y|^{\lambda}}{\left(1-|x|^{2}\right)^{\alpha}\left(1-|y|^{2}\right)^{\lambda-\alpha}} .
\end{aligned}
$$

Here we used the assumption $\alpha \leq \frac{\lambda}{2}$ and the inequality $1-|y| \leq|| x\left|y-x^{\prime}\right|$ for any $x, y \in \mathbb{B}$. Notice that $2^{\lambda-2 \alpha} \leq 2^{\lambda} \leq 2$, which combined with the above results yields

$$
|f(x)-f(y)| \leq n C \frac{|x-y|^{\lambda}}{\left(1-|x|^{2}\right)^{\alpha}\left(1-|y|^{2}\right)^{\lambda-\alpha}}\|f\|_{\mathcal{B}} .
$$


This proves the necessity.

Conversely, suppose that $f$ is hyperbolic harmonic and (3.1) is satisfied. We will show that $f \in \mathcal{B}$. For any fixed $\delta \in(0,1)$, it is known that

$$
|\widetilde{\nabla} f(0)| \leq C \int_{\delta B}|f(a)| d \tau(a) .
$$

Now, replacing $f$ by $f \circ \varphi_{x}-f(x)$ and taking $y=\varphi_{x}(a)$ we get

$$
|\widetilde{\nabla} f(x)| \leq C \int_{E(x, \delta)}|f(x)-f(y)| d \tau(y) .
$$

Therefore,

$$
|\widetilde{\nabla} f(x)| \leq C \sup \{|f(x)-f(y)|: y \in E(x, \delta), x \in B\} .
$$

Note that, for any $y \in E(x, \delta),\left|\varphi_{y}(x)\right| \leq \delta$ and $1-|x|^{2} \simeq 1-|y|^{2}$, so that

$$
\begin{aligned}
\frac{\left(1-|x|^{2}\right)^{\alpha}\left(1-|y|^{2}\right)^{\lambda-\alpha}}{|x-y|^{\lambda}} & \simeq \frac{\left(1-|x|^{2}\right)^{\lambda / 2}\left(1-|y|^{2}\right)^{\lambda / 2}}{|x-y|^{\lambda}} \\
& =\left(\frac{\sqrt{1-\left|\varphi_{y}(x)\right|^{2}}}{\left|\varphi_{y}(x)\right|}\right)^{\lambda} \\
& \geq C .
\end{aligned}
$$

Consequently,

$$
\begin{aligned}
|\widetilde{\nabla} f(x)| & \leq C \sup \left\{\frac{\left(1-|x|^{2}\right)^{\alpha}\left(1-|y|^{2}\right)^{\lambda-\alpha}}{|x-y|^{\lambda}}|f(x)-f(y)|: y \in E(x, \delta), x \in B\right\} \\
& \leq C \sup \left\{\frac{\left(1-|x|^{2}\right)^{\alpha}\left(1-|y|^{2}\right)^{\lambda-\alpha}}{|x-y|^{\lambda}}|f(x)-f(y)|: x, y \in B\right\} .
\end{aligned}
$$

This completes the proof of Theorem 3.1

Theorem 3.2. Let $0<\alpha<\lambda \leq 1$. For any hyperbolic harmonic function $f$ on $\mathbb{B}$, $f \in \mathcal{B}_{0}$ if and only if

$$
\lim _{|x| \rightarrow 1^{-}} \sup \left\{\left(1-|x|^{2}\right)^{\alpha}\left(1-|y|^{2}\right)^{\lambda-\alpha} \frac{|f(x)-f(y)|}{|x-y|^{\lambda}}: y \in \mathbb{B}, y \neq x\right\}=0 .
$$

Proof. Assume that $f \in \mathcal{B}_{0}$ and let $f_{t}(x)=f(t x) \quad(t \in(0,1))$. By (3.1), we have

$$
\left(1-|x|^{2}\right)^{\alpha}\left(1-|y|^{2}\right)^{\lambda-\alpha} \frac{\left|\left(f-f_{t}\right)(x)-\left(f-f_{t}\right)(y)\right|}{|x-y|^{\lambda}} \leq C\left\|f-f_{t}\right\|_{\mathcal{B}}
$$

and

$$
\begin{aligned}
& \left(1-|x|^{2}\right)^{\alpha}\left(1-|y|^{2}\right)^{\lambda-\alpha} \frac{\left|f_{t}(x)-f_{t}(y)\right|}{|x-y|^{\lambda}} \\
& \quad=t^{\lambda} \frac{\left(1-|x|^{2}\right)^{\alpha}\left(1-|y|^{2}\right)^{\lambda-\alpha}}{\left(1-|t x|^{2}\right)^{\alpha}\left(1-|t y|^{2}\right)^{\lambda-\lambda}}\left(1-|t x|^{2}\right)^{\alpha}\left(1-|t y|^{2}\right)^{\lambda-\alpha} \frac{|f(t x)-f(t y)|}{|t x-t y|^{\lambda}} \\
& \quad \leq C \frac{t^{\lambda}}{\left(1-t^{2}\right)^{\lambda}}\left(1-|x|^{2}\right)^{\alpha}\|f\|_{\mathcal{B}} .
\end{aligned}
$$


By the triangle inequality we obtain

$$
\begin{gathered}
\sup \left\{\left(1-|x|^{2}\right)^{\alpha}\left(1-|y|^{2}\right)^{\lambda-\alpha} \frac{|f(x)-f(y)|}{|x-y|^{\lambda}}: y \in \mathbb{B}, y \neq x\right\} \\
\leq C \frac{t^{\lambda}}{\left(1-t^{2}\right)^{\lambda}}\left(1-|x|^{2}\right)^{\alpha}\|f\|_{\mathcal{B}}+\left\|f-f_{t}\right\|_{\mathcal{B}} .
\end{gathered}
$$

In the above inequality, by first letting $|x| \rightarrow 1^{-}$, the first term on the right side converges to 0 , and then letting $t \rightarrow 1^{-}$, the second term on the right side also converges to 0 .

Now suppose that $f$ is hyperbolic harmonic and (3.3) is satisfied. We will show that $f \in \mathcal{B}_{0}$. Fix $r \in(0,1)$. From $(3.2)-(3.3)$ we have

$$
|\widetilde{\nabla} f(x)| \leq C(n, r) \int_{E(x, r)}\left(1-|x|^{2}\right)^{\alpha}\left(1-|y|^{2}\right)^{\lambda-\alpha} \frac{|f(x)-f(y)|}{|x-y|^{\lambda}} d \tau(y) .
$$

By assumption (3.4), for any given $\varepsilon>0$ there exists $\delta \in(0,1)$ such that

$$
\sup \left\{\left(1-|x|^{2}\right)^{\alpha}\left(1-|y|^{2}\right)^{\lambda-\alpha} \frac{|f(x)-f(y)|}{|x-y|^{\lambda}}: y \in \mathbb{B}, y \neq x\right\}<\varepsilon
$$

whenever $|x|>\delta$. Since

$$
\int_{E(x, r)} d \tau=\tau(E(a, r))=\tau(B(0, r))=n \int_{0}^{r} t^{n-1}\left(1-t^{2}\right)^{-n} d t
$$

we have $|\widetilde{\nabla} f(x)|<C \varepsilon$ for any $|x|>\delta$, which means $|\widetilde{\nabla} f(x)| \rightarrow 0$ as $|x| \rightarrow 1^{-}$. This completes the proof

\section{4. $\mathcal{H}$-Besov spaces}

In this section, we give the Holland-Walsh characterization for $\mathcal{H}$-Besov spaces. When $p \rightarrow \infty$, it also reveals the weighted Lipschitz characterization of Bloch spaces.

Theorem 4.1. Let $p \in(2(n-1), \infty)$ and $f$ be hyperbolic harmonic on $\mathbb{B}$. Then $f \in \mathcal{B}_{p}$ if and only if

$$
\int_{\mathbb{B}} \int_{\mathbb{B}}\left(1-|x|^{2}\right)^{\frac{p}{2}}\left(1-|y|^{2}\right)^{\frac{p}{2}}\left(\frac{|f(x)-f(y)|}{|x-y|}\right)^{p} d \tau(x) d \tau(y)<\infty .
$$

To prove this theorem, we need the following

Lemma 4.2. Let $p \geq 1$ and $\alpha>-1$. If $f$ is hyperbolic harmonic on $\mathbb{B}$, then

$$
\int_{\mathbb{B}}\left(\int_{0}^{1} \frac{|\widetilde{\nabla} f(t a)|}{1-t|a|} d t\right)^{p} d \nu_{\alpha}(a) \leq C \int_{\mathbb{B}}|\widetilde{\nabla} f(a)|^{p} d \nu_{\alpha}(a)
$$


Proof. Fix $\varepsilon \in(0,1)$. Observe that for any $t \in[0,1]$ and $a \in \mathbb{B}$ if at least one of $t$ and $|a|$ is less than $\varepsilon$, then $|t a|=t|a|<\varepsilon$, such that $\frac{1}{1-t|a|} \leq \frac{1}{1-\varepsilon}$. Thus the left side in (4.2) can be controlled by

$$
\int_{\mathbb{B}-\varepsilon \mathbb{B}}\left(\int_{\varepsilon}^{1} \frac{|\widetilde{\nabla} f(t a)|}{1-t|a|} d t\right)^{p} d \nu_{\alpha}(a)+C \sup _{x \in \varepsilon \mathbb{B}}|\widetilde{\nabla} f(x)|^{p} .
$$

Denote the first summand above by $I$. From the polar coordinate integral formula and Minkowski's inequality we get

$$
\begin{aligned}
I & =n \int_{\varepsilon}^{1} \int_{\partial \mathbb{B}}\left(\int_{\varepsilon}^{1} \frac{|\widetilde{\nabla} f(t s \zeta)|}{1-t s} d t\right)^{p} d \sigma(\zeta) s^{n-1}\left(1-s^{2}\right)^{\alpha} d s \\
& \leq C \int_{\varepsilon}^{1}\left(\int_{\varepsilon}^{1} \frac{M_{p}(t s,|\widetilde{\nabla} f|)}{1-t s} d t\right)^{p} s^{n-1}\left(1-s^{2}\right)^{\alpha} d s \\
& \leq C \int_{\varepsilon}^{1}\left(\int_{\varepsilon^{2}}^{s} h(\rho) d \rho\right)^{p}\left(1-s^{2}\right)^{\alpha} d s
\end{aligned}
$$

where

$$
h(\rho)=\frac{\rho^{(n-1) / p} M_{p}(\rho,|\widetilde{\nabla} f|)}{1-\rho} .
$$

From Hölder's inequality and Fubini's theorem, we can get the following Hardy's inequality:

$$
\begin{aligned}
\int_{0}^{1}\left(\int_{0}^{s} h(\rho) d \rho\right)^{p}(1-s)^{\alpha} d s & \leq \int_{0}^{1} \int_{0}^{s} h^{p}(\rho) d \rho(1-s)^{\alpha} d s \\
& \leq \int_{0}^{1} \int_{\rho}^{1}(1-s)^{\alpha} d s h^{p}(\rho) d \rho \\
& \leq C \int_{0}^{1} h^{p}(t)(1-t)^{\alpha+1} d t
\end{aligned}
$$

for any $p \geq 1, \alpha>-1$, and $h \geq 0$. As a result,

$$
\begin{aligned}
I & \leq C \int_{0}^{1}\left(\int_{0}^{s} h(\rho) d \rho\right)^{p}(1-s)^{\alpha} d s \\
& \leq C \int_{0}^{1} t^{n-1}(1-t)^{\alpha} M_{p}^{p}(t,|\widetilde{\nabla} f|) d t \\
& =C \int_{\mathbb{B}}|\widetilde{\nabla} f(a)|^{p} d \nu_{\alpha}(a) .
\end{aligned}
$$

It remains to show that $\sup _{\varepsilon \mathbb{B}}|\widetilde{\nabla} f(x)|^{p} \leq C \int_{\mathbb{B}}|\widetilde{\nabla} f(a)|^{p} d \nu_{\alpha}(a)$. For this it is sufficient to prove the inequality

$$
|\widetilde{\nabla} f(x)|^{p} \leq C \int_{E(x, \delta)}|\widetilde{\nabla} f|^{p}(a) d \tau(a) .
$$


Since $f$ is hyperbolic harmonic, we have $f(0)=\int_{\partial \mathbb{B}} f(r \xi) d \sigma(\xi)$ for any $0<r<1$. Replacing $f$ by $f \circ \varphi_{x}$, we see that $f(x)=\int_{\partial \mathbb{B}} f\left(\varphi_{x}(r \xi)\right) d \sigma(\xi)$ for any $x \in \mathbb{B}$ and $0<$ $r<1$. Now we take the gradient about $x$, evaluate at $x=0$, and denote $\psi_{a}(x)=\varphi_{x}(a)$ to get $|\nabla f(0)| \leq C \int_{\partial \mathbb{B}} \mid \nabla\left(f \circ \psi_{r \xi}\right)(0) d \sigma(\xi)$. Since

$$
\left|\nabla\left(f \circ \psi_{r \xi}\right)(0)\right| \leq C\left|\nabla f\left(\psi_{r \xi}(0)\right)\right| \sup _{0<s<1}\left|\nabla \psi_{s \xi}(0)\right|
$$

and $\psi_{r \xi}(0)=\varphi_{0}(r \xi)=r \xi$, it follows that $|\nabla f(0)| \leq C \int_{\partial \mathbb{B}}|\nabla f(r \xi)| d \sigma(\xi)$. Multiplying both sides by $n r^{n-1}\left(1-r^{2}\right)^{-n} d r$ and integrating from 0 to $\delta$, we notice that $|\nabla f(r \xi)| \leq$ $\left(1-\delta^{2}\right)^{-1}|\widetilde{\nabla} f(r \xi)|$ for any $r \in(0, \delta)$ and we conclude

$$
|\nabla f(0)| \leq C\left(1-\delta^{2}\right)^{-1} \delta^{-n} \int_{\delta B}|\widetilde{\tau} f(w)| d \lambda(w) .
$$

If we replace $f$ by $f \circ \varphi_{x}$, then assertion (4.3) follows. This finishes the proof

Proof of Theorem 4.1 Assume that $f \in \mathcal{B}_{p}$. For any $a \in \mathbb{B}$ we have

$$
\frac{|f(a)-f(0)|}{|a|}=\left|\int_{0}^{1} \nabla f(t a) \frac{a}{|a|} d t\right| \leq \int_{0}^{1} \frac{|\widetilde{\nabla} f(t a)|}{1-t|a|} d t .
$$

Therefore, Lemma 4.2 means

$$
\int_{B} \frac{|f(a)-f(0)|^{p}}{|a|^{p}} d \nu_{\alpha}(a) \leq C \int_{B}|\widetilde{\nabla} f(a)|^{p} d \nu_{\alpha}(a) .
$$

Replacing $f$ with $f \circ \varphi_{x}$, integrating with respect to $d \tau(x)$, taking $y=\varphi_{x}(a)$ and setting $\alpha=\frac{p}{2}-n$, we get

$$
\begin{aligned}
\int_{B} \int_{B} & \frac{|f(y)-f(x)|^{p}}{\left|\varphi_{x}(y)\right|^{p}}\left(1-\left|\varphi_{x}(y)\right|^{2}\right)^{\frac{p}{2}} d \tau(x) d \tau(y) \\
& \leq C \int_{B} \int_{B}|\widetilde{\nabla} f(y)|^{p}\left(1-\left|\varphi_{x}(y)\right|^{2}\right)^{\frac{p}{2}} d \tau(x) d \tau(y) \\
& \leq C \int_{B}|\widetilde{\nabla} f(y)|^{p} d \tau(y) \int_{B}\left(1-\left|\varphi_{x}(y)\right|^{2}\right)^{\frac{p}{2}} d \tau(x) \\
& \leq C \int_{B}|\widetilde{\nabla} f(y)|^{p} d \tau(y) .
\end{aligned}
$$

In the last step, we used the estimate $\int_{B}\left(1-\left|\varphi_{x}(y)\right|^{2}\right)^{\frac{p}{2}} d \tau(x) \leq C$ for $p>2(n-1)$, which follows from (2.5) and the Forelli-Rudin estimate in Lemma 2.3. Since

$$
\frac{\left(1-\left|\varphi_{x}(y)\right|^{2}\right)^{\frac{p}{2}}}{\left|\varphi_{x}(y)\right|^{p}}=\frac{\left(1-|x|^{2}\right)^{\frac{p}{2}}\left(1-|y|^{2}\right)^{\frac{p}{2}}}{|x-y|^{p}},
$$

we get (4.1). 
Conversely, supposing that $f$ is hyperbolic harmonic and satisfying (4.1), we will show that $f \in \mathcal{B}_{p}$. For any fixed $\delta \in(0,1)$,

$$
|\widetilde{\nabla} f(x)| \leq C \int_{E(x, \delta)}|f(x)-f(y)| d \tau(y) .
$$

Then, by applying Hölder's inequality and (3.3) with $\lambda=p$ and $\alpha=\frac{p}{2}$,

$$
\begin{aligned}
|\widetilde{\nabla} f(x)|^{p} & \leq C \int_{E(x, \delta)}|f(x)-f(y)|^{p} d \tau(y) \\
& \leq \int_{E(x, \delta)}|f(x)-f(y)|^{p} \frac{\left(1-|x|^{2}\right)^{\frac{p}{2}}\left(1-|y|^{2}\right)^{\frac{p}{2}}}{|x-y|^{p}} d \tau(y) .
\end{aligned}
$$

Thus, (4.1) implies $f \in \mathcal{B}_{p}$. This completes the proof

\section{References}

[1] Ahlfors, L. V.: Möbius Transformations in Several Dimensions. Ordway Professorship Lectures in Mathematics 1981.

[2] Axler, S., Bourdon, P. and W. Ramey: Harmonic Function Theory (Graduate Texts in Mathematics: Vol. 137). New York: Springer-Verlag 1992.

[3] Burbea, J. and S. Y. Li: Weighted Hadamard products of holomorphic functions in the ball. Pac. J. Math. 168 (1995), $235-270$.

[4] Choe, J. S., Kim, H. O. and Y. Y. Park: A Bergman-Carleson measure characterization of Bloch functions in the unit ball of $\mathbb{C}^{n}$. Bull. Korean Math. Soc. 29 (1992), $285-293$.

[5] Erdély, A. et al.: Higher Transcendental Functions, Vol. I. New York: McGraw-Hill 1953.

[6] Hahn, K. T. and E. H. Youssfi: Möbius invariant Besov p-spaces and Hankel operators in the Bergman space on the ball in $\mathbb{C}^{n}$. Complex Variables 17 (1991), 89 - 104.

[7] Holland, F. and D. Walsh: Criteria for membership of Bloch space and its subspace, BMOA. Math. Ann. 273 (1986), 317 - 335.

[8] Nowak, M.: Bloch space and Möbius invariant Besov spaces on the unit ball on $\mathbb{C}^{n}$. Complex Variables 44 (2001), 1 - 12.

[9] Ouyang, C. H., Yang, W. S. and R. H. Zhao: Characterizations of Bergman spaces and Bloch space in the unit ball of $\mathbb{C}^{n}$. Trans. Amer. Math. Soc. 374 (1995), $4301-4312$.

[10] Rainville, E. D.: Special Functions. Bronx - New York: Chelsea Publ. Comp. 1971.

[11] Rudin, W.: Function Theory in the Unit Ball of $\mathbb{C}^{n}$. New York: Springer-Verlag 1980.

[12] Shi, J. H.: Hadamard products of functions holomorphic on a hypersphere (in Chinese). Chinese Ann. Math. Ser. A 9 (1988), 49 - 59.

[13] Stroethoff, K.: Besov type characterization for the Bloch space. Bull. Austral. Math. Soc. 39 (1989), $405-420$.

[14] Stroethoff, K.: The Bloch space and Besov spaces of analytic functions. Bull. Austral. Math. Soc. 54 (1996), $211-219$.

[15] Timoney, R. M.: Bloch functions in several complex variables, Part I. Bull. London Math. Soc. 12 (1980), $241-267$. 
[16] Timoney, R. M.: Bloch functions in several complex variables, Part II. J. Reine Angew. Math. 319 (1980), $1-22$.

Received 11.12.2001 